\title{
LA FORMACIÓN DE MAESTROS PARA UNA ESCUELA INTERCULTURAL EN EL ECUADOR*
}

María Sol Villagómez Rodriguez

\begin{abstract}
Contagiado para siempre de los cantos y los mitos, llevado por la fortuna hasta la Universidad de San Marcos, hablando por vida el quechua, bien incorporado al mundo de los cercadores, visitante feliz de grandes ciudades extranjeras, intenté convertir en lenguaje escrito lo que era como individuo: un vínculo vivo, fuerte, capaz de universalizarse, de la gran nación cercada y la parte generosa, humana, de los opresores. El vínculo podía universalizarse, extenderse; se mostraba un ejemplo concreto, actuante. El cerco podía y debía ser destruido; el caudal de las dos naciones se podía y debía unir. Y el camino no tenía por qué ser, ni era posible que fuera únicamente el que se exigía con imperio de vencedores expoliadores, o sea: que la nación vencida renuncie a su alma, aunque no sea sino en la apariencia, formalmente, y tome la de los vencedores, es decir que se aculture. Yo no soy un aculturado; yo soy un peruano que orgullosamente, como un demonio feliz habla en cristiano y en indio, en español y en quechua
\end{abstract}

José María Arguedas

\section{Introducción}

La independencia de España y la creación del Estado Nación ecuatoriano, significó un deslindamiento político y económico de los colonizadores españoles, pero no significó una real descolonización. La colonialidad del saber, del ser y del poder (...) se mantiene hasta la actualidad; tal como menciona (WALSH, 2009, p. 30) "para los pueblos negros, como para los indígenas y mestizos, la colonialidad ha operado a nivel intersubjetivo y existencial, permitiendo la deshumanización de algunos, la sobrehumanización de otros y la negación de los sentidos integrales de la existencia y humanidad".

"DOI - 10.29388/978-65-86678-84-0-0-f.139-156 
El sistema escolar ha sido una de las herramientas de colonización, incluso hasta nuestros días, por ello, cuando nos referimos a una escuela colonial y colonizadora, hacemos referencia en realidad a un sistema educativo que puede ser visto como instrumento de colonización. Así, se puede afirmar que la institución escolar ha sido de carácter civilizatoria, homogénea y monocultural; destinada a servir al proyecto de estado nación blanco-mestizo y como tal, al desconocimiento y negación de las diferencias y en consecuencia monocultural; como tal ha colocado el conocimiento y la lengua occidental en el centro de los procesos educativos, "descartando por completo la producción intelectual indígena y afro" (WALSH, 2009, p. 30), y cuya intención ha sido, la formación de una identidad nacional única.

$\mathrm{Ni}$ la Europa occidental colonialista, moderna y racional, ni la sociedad criolla blanco/mestiza, reconocieron en aquellos 'otros' la capacidad de producir conocimientos válidos, universales. Desde los primeros momentos del proceso de colonización, los saberes que habrían de ser institucionalizados y aprendidos por indígenas y negros fueron definidos al margen de sus intereses. En consecuencia, la escuela, cuando llegó, lo hizo para 'incorporar', para conducir el tránsito de estas poblaciones hacía la sociedad 'civilizada' (ROJAS; CASTILLO, 2005, p. 138).

Como contraparte a los sistemas de educación colonial, surgen las iniciativas de los pueblos olvidados y relegados de la historia, con su propuesta de una educación que reivindique su cultura, que los reconozca como parte de la historia y que contribuya a la construcción de una sociedad donde las diferencias quepan. Nos referimos a la educación intercultural que, como esfuerzo descolonizador posibilitaría la incorporación de otras formas de pensamiento, otras formas de enseñar y aprender, otros conocimientos y saberes y, otras lenguas, en los sistemas escolares, más allá de los conocimientos occidentales, homogeneizadores y coloniales.

El texto inicia con la presentación de la trayectoria de la Educación Intercultural Bilingüe (EIB) en el Ecuador y sus aportes para interculturalización de la escuela ecuatoriana. A continuación nos referimos a la relación "universidad-escuela básica" desde la experiencia de formación de docentes para la escuela intercultural de la Carrera de EIB de la Universidad Politécnica Salesiana del Ecuador (UPS), para finalmente 
aportar a la reflexión de cuáles son los docentes que la escuela intercultural bilingüe requiere.

\section{Trayectoria de la educación intercultural bilingüe en el Ecuador}

Tanto en la organización indígena como en los académicos, existe el consenso de que las primeras escuelas para la EIB fueron las escuelas indígenas de Cayambe, creadas en los años de 1940 por Dolores Cacuango, dirigente indígena kichwa quien vinculada a la Federación Ecuatoriana de Indios (FEI), lidera un proyecto de creación de escuelas en las haciendas donde los indígenas vivían en situación de servidumbre. Este proyecto es emblemático porque, como se ha dicho, marca el inicio de procesos de "educación propia". Entre los rasgos más innovadores de las escuelas indígenas de Cayambe, se destaca la impartición de las clases en lengua kichwa y no en castellano; su enfoque es de corte emancipador en tiempos donde la población indígena estaba sujeta al sistema de explotación hacendatario $\mathrm{y}$, como tales constituyen espacios fundamentales para la organización y la reivindicación de los derechos de los pueblos indígenas.

Otra experiencia relevante, son las Escuelas Radiofónicas de Educación Popular (ERPE), propuesta educativa que surge en la provincia de Chimborazo en la sierra central del Ecuador. Este proyecto educativo fue emprendido en 1963, por Monseñor Leonidas Proaño, religioso católico, conocido también como "obispo de los indios". ERPE era un proyecto de alfabetización en el cual el docente, un monitor indígena y comunero, que tenía conocimientos escolares, estaba encargado de acompañar los aprendizajes de los estudiantes en lengua indígena. Este programa, también de corte popular y liberador, ofrecía además de la alfabetización contenidos para el trabajo (MOYA, 1987, p. 404). El impacto de estas escuelas fue relevante para la educación indígena del país, las cifras nos muestran que entre 1963 año en que fueron reconocidas legalmente y 1972, las ERPE alfabetizaron a unos 13.000 campesinos adultos de las provincias de la sierra, costa y el oriente (CHIODI, 1990, p. 357); Por su énfasis liberador, al igual que las escuelas de Cayambe, la escuelas indígenas de Chimborazo fueron relevantes en su apoyo a la organización indígena de la provincia y por su lucha por la educación y por la tierra. 
El Sistema de Educación Radiofónica Bicultural Shuar (SERBISH), creado en 1973 (MOYA, 1987, p. 394), creado para atender las demandas educativas de los Shuar, pueblo indígena de la amazonia ecuatoriana, fue emprendido con el apoyo de la misión salesiana en territorio. Como proyecto educativo es relevante porque para los procesos formativos se empleaba el shuarchicham (idioma del pueblo shuar) y el castellano (MOSEIB, 2013, p. 15), además en términos de legado a la educación, este programa ofreció un importante aporte al país porque propició el reconocimiento de la biculturalidad de la educación (TORRES, 1992, p. 15). Por otro lado, la relevancia de este programa, al igual que los antes expuestos, se encuentra en que propicia un proceso organizativo del pueblo indígena, en este caso de la comunidad Shuar (CHIODI, 1990, p. 358).

La Fundación Runacunapac Yachana Huasi, que en lengua kichwa significa escuela para indígenas, fue creada en 1972 en Simiatug, en la provincia de Bolívar, zona de alto páramo de la sierra central del país. Este proyecto, delineó una propuesta de educación indígena donde "el kichwa fue el idioma central en la enseñanza, los maestros eran kichwa hablantes y miembros activos de la organización, quienes no sólo ejercían la labor de maestros sino también diferentes actividades que requería la comunidad" (GONZÁLEZ, 2011, p. 40). Este proyecto, también se caracterizó por el rechazo a la escuela tradicional hispana por considerarla distante a la realidad y necesidades de la comunidad. Al igual que otras experiencias mencionadas antes las escuelas se articulan con la organización indígena y constituye un referente importante para la EIB del país (VILLAGÓMEZ, 2016).

El Sistema de Escuelas Indígenas de Cotopaxi (SEIC) se trató de una iniciativa de educación propia que surge en el año de 1975 en las parroquias de Zumbagua y Chugchilán en la provincia de Cotopaxi en la sierra central. Este proyecto fue impulsado por la organización comunitaria y contó con el apoyo de la misión salesiana. Las escuelas del SEIC se caracterizaron por educación en lengua indígena, pero además por el desarrollo de un pensamiento propio alineado al proyecto político del pueblo indígena de Cotopaxi, la lucha por sus derechos y por la tierra (GRANDA, 2016).

Más adelante, entre 1980 y 1984, en el marco del Proyecto Nacional de Alfabetización del Ecuador, el Ministerio de Educación elabora el Subproyecto de alfabetización en kichwa, dirigido específicamente a población indígena kichwa. Su importancia radica, no solo por la atención a 
un importante número de la población indígena con un modelo educativo en lengua y cultura propia, sino por su vínculo con la academia. Este proyecto fue ejecutado por el Centro de Investigaciones para la Educación Indígena (CIEI) de la Pontificia Universidad Católica del Ecuador (PUCE) por delegación del Ministerio de Educación y Cultura (MOYA, 1987, p. 396). El aporte más notable del programa fue el reconocimiento de la necesidad de la especificidad educativa de los pueblos indígenas. Al tratarse de un programa estatal con participación directa de los indígenas, contribuyó a la visibilización de las necesidades educativas de los pueblos, además de propiciar la generación política educativa diferenciada para la población indígena. El programa formó también a importantes líderes e intelectuales indígenas.

Estos y otros programas de educación indígena constituyen el antecedente fundamental para el posterior reconocimiento de la Educación Intercultual Bilingüe en el Ecuador (EIB) y la creación de la Dirección Nacional de Educación Intercultural Bilingüe (DINEIB) en noviembre de 1988 como una dependencia del Ministerio de Educación del Ecuador, vinculada a la CONAIE (Confederación de Nacionalidades Indígenas del Ecuador), con autonomía técnica, administrativa y financiera (CONEJO, 2008, p. 70) y la posterior elaboración del (MOSEIB) Modelo del Sistema de Educación Intercultural Bilingüe (Registro Oficial N. 278 de 17 septiembre del 1993).

Sin duda, tanto la creación de la DINEIB, como la publicación del MOSEIB, constituyen uno de los logros más relevantes en el campo de la educación del movimiento indígena del país organizados en la CONAIE, con una amplia incidencia en la política educativa para pueblos indígenas y clara demostración de su enorme capacidad de resistencia. Así, "la EIB era la respuesta a una necesidad de generar una educación cuyos valores enfrenten a la "moderna colonización" (OVIEDO, 2017, p. 55). Según señala el líder indígena (CONEJO, 2008)

La educación Intercultural Bilingüe es una propuesta de vida, por la vida, en la vida, para la vida de los pueblos y las nacionalidades del Ecuador. Responde al ideal de construir un Estado plurinacional, y una Sociedad intercultural y multilingüe (p. 79) 


\section{El Modelo del Sistema de la Educación Intercultural Bilingüe (MOSEIB)}

El modelo para la educación intercultural bilingüe del país se plasma en un documento de tipo curricular referencial para el trabajo educativo en las instituciones escolares que atienden a los pueblos y nacionalidades indígenas. En su justificación el (MOSEIB, 2013) plantea la necesidad de recuperar formas de educación ancestral, familiar y comunitaria $(p, 26)$, fundamentadas en los siguientes principios ( $p .28)$, que en suma destacan la importancia de los valores culturales y familiares, las lenguas y conocimientos como base de la educación y de una docencia respetuosa:

- respeto y cuidado a la Madre Naturaleza; la persona, su familia y la comunidad son los actores principales del proceso educativo;

- la formación de las personas se inicia desde la EIFC y continúa hasta el nivel superior. Perdura a lo largo de toda la vida. Los padres deben prepararse desde antes de engendrar a la nueva persona;

- la lengua de las nacionalidades constituye la lengua principal de educación y el castellano tiene el rol de segunda lengua y lengua de relación intercultural;

- el currículo debe tener en cuenta el Plan de Estado plurinacional, el modo de vida sustentable, los conocimientos, prácticas de las culturas ancestrales y de otras del mundo;

- los aspectos: psicológicos, culturales, académicos y sociales en función de las necesidades de los estudiantes;

- las maestras y los maestros son profesionales de la educación, manejan pluralidad de teorías y prácticas, por lo tanto, no dependen de esquemas homogeneizantes (p.28).

El documento presenta además algunas estrategias de implementación, de tipo pedagógico para su utilización en los contextos escolares vinculadas a las necesidades educativas de las diferentes nacionalidades del Ecuador; sus formas de aprender y las pedagogías propias; así como la revalorización, reproducción y vitalidad de la cultura, saberes y lengua. Algunas de las más relevantes son las siguientes: 
(...) desarrollar los saberes, sabidurías, conocimientos, valores, principios, tecnologías y prácticas socio culturales y sistemas cosmovisionales en relación al entorno geobiológico y socio-cultural, usando las lenguas ancestrales; elaborar y aplicar el calendario vivencial de la nacionalidades en el proceso educativo; desarrollar los valores éticos, científicos, estéticos y lúdicos; constituir a los $\mathrm{CECIBs,}$ en espacios de desarrollo de conocimientos colectivos sobre recursos genéticos, diversidad biológica y agro-biodiversidad, así como, de los saberes comunitarios, la formación técnico-científica y la promoción de las diversas formas de desarrollo productivo y cultural; (...) incluir en los contenidos curriculares: el espacio matemático de representación, los esquemas lógicos y los sistemas de clasificación de las nacionalidades; incorporar al currículum la historia y las manifestaciones artísticas de los pueblos y nacionalidades y de otras culturas del mundo; aplicar una metodología de aprendizaje que tome en cuenta las prácticas educativas de cada cultura y los avances de la ciencia; (...) elaborar material audio-visual para los centros educativos y organizar bibliotecas comunitarias; adaptar las actividades educativas al horario y calendario social de las comunidades sin perjudicar la normativa establecida; facilitar el aprendizaje de otras lenguas y culturas para mejorar las relaciones comunicativas; respetar el ritmo de aprendizaje y la organización de las modalidades curriculares, según las necesidades de las nacionalidades;(...) (p.32, 33).

EI MOSEIB es un documento de relevancia no solo para la educación indígena del país, sino para la educación en general. La educación de todos los ecuatorianos no debería desestimar la experiencia de la educación indígena y la incorporación complementaria del los saberes, conocimientos, historia y lenguas indígenas.

\section{La interculturalidad en el currículo del sistema educativo ecuatoriano}

Uno de los principales aportes de la educación intercultural bilingüe al país fue el colocar sobre el debate de la educación en general la necesidad de incluir la interculturalidad como un contenido curricular para toda la sociedad ecuatoriana. Hay que aclarar en este punto que en el Ecuador existen dos currículos por un lado esta el MOSEIB, destinado a la educación de los pueblos y nacionalidades indígnenas, cuyas características 
generales ya hemos mencionado en el acápite anterior y por otro el Currículo general de los Niveles de Educación obligatoria, denominado también currículo hispano, destinado a la población estudiantil blanco mestiza de país.

A partir de la década de 1990, el currículo generla incluye la interculturalidad, como eje tranversal o como contenido educativo. Así, la primera vez que se incluye la interculturalidad en el currículo general hispano de educación básica, fue en el documento de 1996. En este, la interculturalidad se plantea como eje transversal, vista como "un proceso de construcción de una condición que permitirá en el futuro equilibrar las posibilidades para sectores de la población históricamente desfavorecidas" (MINISTERIO DE EDUCACIÓN, 1996, p. 122) y con el fin de "generar una actitud de respeto hacia los diversos grupos socio-culturales y la eliminación de caducos esquemas discriminatorios en favor de igualdad de oportunidades" (MINISTERIO DE EDUCACIÓN, 1996, p. 122).

En 2010, se expide la LOEI (Ley Orgánica de Eduación Intercultural), este documento jurídico "...determina los principios y fines generales que orientan la educación ecuatoriana en el marco del Buen Vivir, la interculturalidad y la plurinacionalidad..." (LOEI, Art. 1). (MINISTERIO DE EDUCACIÓN, 2010a). La LOEI reconoce todo el sistema educativo nacional como intercultural, por tanto la educación en general tiene un enfoque intercultural. Sin embargo, en la práctica se avanza solamente a una revisión y ajuste curricular y como resultado de este proceso, el Ministerio de Educación, publica un documento denominado Actualización y Fortalecimiento curricular para la Educación General Básica. En este documento se incluye por primera vez en el sumak kawsay o buen vivir, concebido como el eje rector de la transversalidad del currículo en el marco del cual se plantean algunos temas para la educación escolar. La interculturalidad es una de estas temáticas transversales que se encuentran incluidas dentro del principio rector del buen vivir. Para la política educativa, el buen vivir y la educación interactúan en doble vía, como derecho a la educación y, como una educación para la sociedad del buen vivir (MINISTERIO DE EDUCACIÓN, 2010b, p. 16). Finalmente, la interculturalidad se incluye como temática principalmente en la asignatura de estudios sociales.

Finalmente, en 2016, se lleva a cabo una nueva revisión del currículo nacional por parte del Ministerio de Educación, como consecuencia de esta revisión, se elabora un documento denominado 
Currículo de los niveles de Eduación Obligatoria. Este documento ya no plantea la interculturalidad de manera transversal, pero lo incluye como contenido y como objetivo en algunas áreas curriculares y asignaturas, principalmente en ciencias sociales, en los contenidos de convivencia, de historia y de ciudadanía.

Aunque como afirman algunos autores (GRANDA, 2007; VILLAGÓMEZ, 2017) en estos documentos la inclusión de la interculturalidad puede interpretarse como aditiva y relacionada más desde una perspectiva de tolerancia y respeto a la diferencia, más propio de un enfoque multiculturalista que intercultural, su tratamiento en el currículo y en la ley de educación ya es un avance importante, porque abre la posibilidad de una educación que incorpore otras lenguas, otros conocimientos y otros saberes, además del conocimiento occidental, blanco. Corresponde a las escuelas y a los educadores contribuir con estrategias adecuadas, contextualizadas y pertinentes a las necesidades educativas acordes a la diversidad de la población ecuatoriana para aportar a la construcción de la sociedad del buen vivir, anhelo histórico de los pueblos originarios del Ecuador.

Corresponde también a las escuelas y a los maestros incorporar en su trabajo educativo la lucha constrante contra el racismo, la discriminación y la exclusión y a favor de la ateridad y de la cooperación y de valoraciones mutuas.

\section{La experiencia de la Carrera de EIB de la UPS y los desafíos de la formación de docentes para la escuela intercultural.}

La formación docente para la educación intercultural, es una necesidad del Ecuador como sociedad en su conjunto y aunque, si bien las principales iniciativas surgen específicamente direccionadas y por las demandas y luchas de los pueblos indígenas; de sus propuestas y aciertos, se pueden desprender importantes aprendizajes que permiten aportar a la formación de un profesorado que pueda aportar a la construcción de una escuela intercultural y decolonial para todos los ecuatorianos.

Ya se ha señalado más arriba que la institucionalización de la educación intercultural bilingüe, fue uno de los logros más relevantes de la lucha indígena de fines de la década de 1980 y de inicios de la década de 1990 en el Ecuador. Este proceso exigió además de la autornomía del 
sistema intercultural bilingüe, el contar con profesionales de la educación formados para ejercer la docencia en sus propias escuelas.

Así, la demanda de formación docente para la escuela intercultural bilingüe exigía, contar con instituciones de educación superior para formar a estos docentes y con programas alternativos en relación con las necesidades de los sujetos y de los contextos.

A continuación, presentamos la experiencia de la Carrera de Educación Intercultural Bilingüe de la UPS, uno de los proyectos educativos dirigido a pueblos indígenas más emblemático y con mayor durabilidad en el tiempo del país, al permanecer vigente por más de 25 años.

En 1994, por la demanda de la MIC (Movimiento Indígena de Cotopaxi) y con el apoyo de la Misión Salesiana de Zumbagua y de la Universidad Politécnica Salesiana, nace el PAC (Programa Académico Cotopaxi), actualmente Carrera de Educación Intercultural Bilingüe de la UPS. El programa se crea en territorio indígena, en el páramo del Quilotoa en la Provincia de Cotopaxi, contexto en el cual desde hace varias décadas venía operanado el SEIC, como ya se ha señalado anteriormente.

La mayor parte de los primeros estudiantes del PAC fueron alfabetizadores y maestros del SEIC. "Para los alumnos-maestros que se encontraban en el ejercicio de la docencia en las esuelas comunitarias, el PAC constituyó una importante oportunidad para su profesionalización" (VILLAGÓMEZ, 2019a, p. 220).

Actualmente, la Carrera de EIB cuenta con más de 26 años de funcionamiento ininterrumpido, durante este tiempo, se ha extendido a otras provincias del país, por demanda de los propios pueblos indígenas de cada zona. Actualmente, la carrera funciona en Otavalo, Cayambe, Latacunga, Riobamba y Wasakentza, atendiendo principalmente a población kichwa andina, achuar amazónica, campesinado y pueblo mestizo, viculado a la educación intercultural. A la fecha ha graduado más de 1000 maestros (UPS, 2019).

En esta experiencia, se destaca la especificidad, la búsqueda de pertenencia, la cercanía con la comunidad y organización indígena, el trabajo en territorio y el desafío por mantener una oferta educativa próxima a los requerimientos de la educación intercultural.

Como proyecto educativo dirigido a la formación de profesores para la EIB, se caracteriza por ser una propuesta alternativa y antihegemónica que intenta responder a la realidad de exclusión y desigualdad que los 
pueblos indígenas, campesinado y sectores populares han enfrentado en el país durante más de cinco siglos.

Así, el proyecto educativo incluye elementos que de manera explícita muestran una intencionalidad educativa de liberación de los estudiantes y de sus pueblos. Según lo planteado en la justificación de dicho proyecto (UPS, 1995) (UPS, 2005), el PAC es visto como un instrumento de a) liberación, b) autoconciencia y autovaloración, c) inspirado en la vida comunal, d) superarción de la pobreza; e) valoración de los saberes y conocimientos propios y de acercamiento al conocimiento occidental; f) constructor de interculturalidad $y, g$ ) que propicia el fortalecimiento del bilinguismo.

Concomitantemente con lo expuesto el objetivo general de la carrera en el último rediseño curricular (UPS, 2017) se dirige a la formación de profesionales de la educación capaces de diseñar y desarrollar propuestas educativas con pertinencia social, cultural y lingüística para contribuir al desarrollo de los pueblos y nacionalidades indígenas y a la construcción de una sociedad intercultural. En la formación de profesorado se vislumbra la contribución al logro de la interculturalidad, como proyecto de país.

Las asignaturas que se desarrollan en el proyecto formativo involucran contenidos educativos que propician el conocimiento y la valoración de la historia, cultura y organización de los pueblos; los saberes y conocimientos propios y ancestrales; el fortalecimiento del bilinguismo (kichwa- español o achuar- español, dependiendo del contexto). Algunas de las asignaturas más representativas son (UPS, 2005) (UPS, 2017): Cosmovisión indígena; Historia de los pueblos y nacionalidades indígenas; Modelos y experiencias de la educación intercultural bilingüe; Lengua indígena; Educación y Bilinguismo; Pedagogías Intercultural, por citar algunas más específicas a su formación, junto con otras más clásicas de la formación docente

Según lo expuesto en trabajos anteriores sobre este mismo tema (VILLAGÓMEZ, 2016; VILLAGÓMEZ, 2019), la implementación del proyecto educativo y su gestión tiene algunos rasgos que resulta interesante revisar, sobre todo por el carácter creativo de la propuesta en el afán de dotar de pertinencia al intentar responder a las necesidades de los destinatarios, principalmente indígenas comuneros y campesinado: 
- La carrera se lleva a cabo en territorio, en las provincias y poblaciones donde la UPS cuenta con centros de apoyo y docentes que llevan adelante la tarea de acompañar el proceso formativo de los alumnos-maestros.

- Un proyecto educativo dialogado con los destinatarios. La presencia en territorio permite un diálgo no solo con los estudiantes, sino además con la organización indígena. Los vínculos con la organización indígena, tanto a nivel macro, como con las organizaciones comunitarias de los territorios donde esta tienen incidencia, tal commo se puede deducir del testimonio de uno de los coordinadores-gestores de la carrera:

Los coordinadores de los centros de apoyo son los actores que vienen de una red que también son claves en lo que sería una relación orgánica entre carrera y organizaciones y comunidad (...) tienen excelentes relaciones con las organizaciones, con los gobiernos locales, pero también con los distritos, municipios. Son redes invisibles que dan vida al proyecto, son fomas propias de organización andina y que en definitiva ayudan a mantener contacto con tierra, ayuda a alimentar la carrera siempre. No entenderías el éxito de la carrera y la pertinencia sin estas relaciones más informales (...) (Profesor B, entrevista, en VILLAGOMEZ, 2016).

- Los procesos educativos se acompañan con medios y recursos diversificados, dependiendo de las condiciones geográficas y de accesos de proveniencia de los estudiantes, entre ellos, ambientes virtuales de aprendizaje, la elaboración de guías de estudio, materiales digitales y materiales impresos. Se priorizan uno u otros dependiendo de la ubicación geográfica de los estudiantes y las condiciones de conectividad.

Del diálogo con los actores del proyecto educativo, se desprenden algunos desafíos para la formación de profesorado para una escuela intercultural. Entre los aspectos más relevantes destaca la necesidad de a los maestros las herramientas para: (VILLAGÓMEZ, 2016) (VILLAGÓMEZ, 2019b).

- Conocer las características del contexto histórico, social, cultural, político y econónico donde ocurre el hecho educativo y la necesidad de interrogar las inequitativas relaciones de poder entre pueblos indígenas, afrodescendientes y blanco-mestizos que han configurado al Ecuador. -El reconocimiento de la educación como posibilitadora de transformación para la construcción de una sociedad igualitaria, intercultural para el logro 
del Buen Vivir y que propicie el ejercicio de una docencia crítica y comprometida.

- Fomentar la autovaloración y el fortalecimiento identitario o lo que implica el trabajo para el aprecio de la lengua y la cultura propia, el autorreconocimiento y el orgullo étnico desde el conocimiento de la propia historia, su lugar y aporte en la historia del país; la participación en el proyecto histórico de su propio pueblo y como miembro de una comunidad que tiene objetivos comunes.

- Fomentar el diálogo de saberes, la inclusión y vitalidad de conocimientos propios y ancestrales; su valoración e inclusión en los procesos educativos; posibilitando el desarrollo de estrategias que favorezcan la complementariedad su complementariedad con el conocimiento occidental. El dominio, uso y vitalidad en la vida cotidiana y en la vida escolar de la lengua propia y de la lengua occidental propiciando así el logro del bilingüismo.

Al ser docente indígena no dejamos a que desvincule de la cultura propia a los chicos, eso es más importante, porque si los chicos desvinculan de su cultura, estamos fregados, porque puede ir desapareciendo la comunidad. (JV, 2017) (IZA et al., 2019).

- La sistematización y organización de conocimientos y saberes su pedagogización y el diseño de estrategias didácticas elaboración de recursos educativos para el trabajo escolar y de aula. Junto con ello, indagar desde los saberes, las costumbres y modos propios de educar para mejorar la práctica docente.

- El diseño de proyectos educativos y curriculares cultural y lingüísticamente pertinentes y que propicien la práctica de la interculturalidad, la reflexión y la crítica.

- Desarrollar pedagogías interculturales y descolonizadoras; pedagogías "otras" (ARROYO, 2012) (VILLAGÓMEZ, 2018) pedagogías insumisas (MEDINA, 2015) que parten de las necesidades educativas de los sujetos que se educan, entendidas como la praxis educativa que interroga los modos de educación tradicional, monocultural y eurocéntrico y que generen los espacios y los mecanismos para que "otra educación" sea posible.

- Luchar y prevenir la discriminación racial, de género, el machismo, el racismo y el clasismo y para desarrollar propuestas y procesos educativos 
incluyentes y de equidad. Junto con ello, valorar la docencia de la educación básica como una actividad principalmente femenina y con ello el aporte de las mujeres y mujeres indígenas a la educación de sus propios pueblos. En la Carrera de EIB, más del 70\% del alumnado es femenino (UPS, 2018a) ; y en el país más del $71 \%$ del profesorado de educación básica es femenino (MINISTERIO DE EDUCACIÓN, 2018-2019).

- Investigar su propia práctica docente, interrogarla y mejorarla. El desarrollo de la autocrítica y de la creatividad. Una docencia colaborativa y de aprendizajes mutuos y de interrelación y cooperación con la diferencia.

- Comprender a la educación, como un proyecto afectivo, "que implica el conocimiento del otro y de su realidad y el aprendizaje del otro, de aprendizajes mutuos" (VILLAGÓMEZ, 2016, p. 291). Una educación en alteridad "(...) para que ellos salgan de un mundo y puedan conocer lo otro". (AG, 2017) (IZA et al., 2019).

\section{Consideraciones finales}

Por su enorme potencial descolonizador, la educación intercultural ha contribuido desde sus orígenes a la construcción, no solamente de una escuela para todos, sino de una escuela decolonial. La inclusión en la educación no solo de sectores de la población históricamente relegados, sino además de sus conocimientos, su cultura y sus lenguas es un aspecto de gran relevancia a la hora de pensar en una educación que atienda a todos los sectores del país.

Se trata de una escuela propuesta por los propios pueblos, vinculada a sus demandas más auténticas y que como tal aporta a las mismas comunidades, su desarrollo y su participación en la sociedad en su conjunto. La "escuela propia", como se conoce a este tipo de experiencias da luego origen a todo un sistema de educación intercultural bilingüe para los pueblos indígenas y aporta de manera significativa a la reflexión y posterior inclusión de la dimensión interculturalidad en la educación general del país.

Esta escuela intercultural bilingüe o que incluye la dimensión interculturalidad, requiere docentes que puedan responder a las necesidades educativas de los sujetos que se educan y de sus contextos y a uno de los anhelos más profundos de la educación que es la construcción de una sociedad más equitativa, más justa, incluyente e intercultural. En 
suma una sociedad del "Sumak Kawsay" o del Buen Vivir, como los soñaron nuestros antepasados.

La formación de este profesorado es uno de los mayores desafíos y así el sistema educativo nacional ha tenido que aprender del sistema del sistema intercultural bilingüe, probablemente la formación de docentes para una escuela que pueda incluir de manera real la dimensión interculturalidad, podrá aprender de las experiencias de formación de docentes para la EIB, como es el caso de Carrera de EIB de la UPS que se expone en este artículo.

¿Qué caracteriza a un docente para una escuela intercultural?, ¿Cómo debe ser la formación de ese profesorado?, son interrogantes sobre las cuales es necesario seguir reflexionando, investigando y aprendiendo de las prácticas existentes.

Pensar en una docencia y en una escuela intercultural que pueda incluir la diferencia. Pensar en que las diferencias culturales, lingüísticas, epistémicas puedan ser complementarias en el sistema educativo y no contrapuestos; donde el principio de la alteridad constituya un eje central de la educación, siguen siendo desafíos importantes para la educación y la escuela ecuatoriana. Sin embargo hay camino trazado, los maestros tenemos la responsabilidad histórica de avanzar.

\section{Referencias}

AG. Entrevista Mujeres Indígenas . (GIEl, Entrevistador), 2017

ARROYO, M. Outros sujeitos outras pedagogías. Belo Horizonte: Editorial Vozes, 2012.

CHIODI, F. La Educación Indígena en América Latina. Quito: EBI (MEC-GTZ) \& ABYA-YALA, 1990.

CONEJO, A. Educación Intercultural Bilingue en el Ecuador. La propuesta educativa y sus procesos. Alteridad, [s.I.], v. 3, n $2,2008$.

GONZÁLEZ, M. Movimiento indígena y educación intercultural en el Ecuador. México: Universida Autónoma de México-CLACSO, 2011. 
GRANDA, $S$, et al. Logros y retos de la eduación intercultural para todos en el Ecuador. Quito PREOIB-ANDES, UPS, 2007

GRANDA, S. Estado, educación y pueblos indígenas en los Andes ecuatorianos. Alteridad. Revista de Educación, [s.I.], v. 11, n 2, p. 221-230, 2016.

IZA, A. et al. Las mujeres indígenas podemos salir adelante. Retratos y relatos de mujeres indígenas universitarias. Quito: Abya-Yala, 2019.

MEDINA, P. Pedagogías insumisas. Movimientos político-pedagógicos y memorias colectivas de educaciones otras en América Latina. México: Juan Pablos Editor, 2015.

MINISTERIO DE EDUCACIÓN. Ministerio de Educación. Quito: [s.n.], 1996.

MINISTERIO DE EDUCACIÓN. LOEI. Quito: [s.n.], 2010a. Disponível em: https://bit.ly/2KENvrz.

MINISTERIO DE EDUCACIÓN. MINISTERIO DE EDUCACIÓN. Quito: [s.n.], 2010b.

MODELO DEL SISTEMA DE EDUCACIÓN INTERCULTURAL BILINGUE MOSEIB. MOSEIB. Quito: [s.n.], 2013. Disponível em: https://goo.gl/76EUyc.

MOYA, R. Educación Bilingüe en el Ecuador: Retos y Alternativas. INDIANA, [s.I.], n 11, p. 387-406, 1987.

OVIEDO, A. Educación Intercultural Bilingüe en el Ecuador (1989-2007): Voces. Quito: [s.n.], 2017. Disponível em: https://bit.ly/3bLH9m9.

ROJAS, A.; CASTILLO, E. Educar a los otros. Estado, políticas educativas y diferencia cultural en Colombia. Popayán: Editorial Universidad Del Cauca, 2005.

TORRES, V. La escuela India ¿̇ntegración o afirmación étnica? In: TORRES, V. (Org.). Quito: Abya-Yala, 1992. 
UPS-SGU. Sistema General de la UPS. Cuenca: [s.n.], 1995.

UPS. Sistema Genral de la UPS. Cuenca: [s.n.], 2005.

UPS. Sistema General de la UPS. Cuenca: [s.n.], 2017.

UPS. Educación Intercultural Bilingüe. 2018a. Disponível em:

https://www.ups.edu.ec/educacion-intercultural-bilingue-quito. Acesso em: 29 nov. 2018.

UPS. Informe de graduados. Quito: [s.n.], 2018b.

VILLAGÓMEZ, M. S. Práctica de la Interculturalidad, descolonización y formación docente: El Programa Académico Cotopaxi del Ecuador. Universidad Federal de Minas Gerais, 2016.

VILLAGÓMEZ, M. S. Educación Intercultural en el currículo nacional, desafíos para la formación y el trabajo docente. La formación y el trabajo docente en el Ecuador. Quito: Editorial Abya-Yala, 2017.

VILLAGÓMEZ, M. S. "Otras Pedagogías": La experiencia de la Carrera de Educación Intercultural Bilingue-UPS. Alteridad. Revista de Educación, [s.I.], v. $13, n^{\circ} 1$, p. 30-41, 2018. DOI:

https://doi.org/10.17163/alt.v13n1.2018.02.

VILLAGÓMEZ, M. S. Desafíos de la formación de profesorado para la educación intercultural bilingüe. Interculturalidad. Problemáticas y perspectivas diversas. Quito: Editorial Universitaria Abya-Yala, 2019a.

VILLAGÓMEZ, M. S. Profesoras indígenas. Aportes de la educación superior intercultural bilingüe a la formación docente. Políticas Públicas, autonomía y participación docente en contextos de transformación y cambio. Cuenca: Abya-Yala, 2019b.

WALSH, C. Interculturalidad, Estado, sociedad. Luchas (de) coloniales de nuestra época. Quito: Abya-Yala, 2009 